\title{
The Life Cycle of Dwarf Stentors
}

\section{Benjamin J. Scherlag PhD}

The Department of Medicine, The Heart Rhythm Institute, The University of Oklahoma Health Sciences Center, Oklahoma City

*Corresponding author: Benjamin J. Scherlag PhD, 1200 Everett Drive (6E103), Oklahoma City, OK. 73104,

E-mail: benjamin-scherlag@ouhsc.edu

\begin{abstract}
While studying the mode of encysting shown by large forms of the unicellular animal of the genus Stentor (500 - 1000 microns) we were able to consistently demonstrate the appearance of dwarf forms, not previously described, which arose from nuclei released from the disrupted larger cells. These larger cells had encysted in an unfavorable environment due to evaporation to a dry state. When sterile Stentor media was added to the deep well slides, active micronuclei were observed undergoing segmentation and separation into 2 or 4 dwarf Swimmers. Following the progression of these dwarfs over several days, allowed us to view their life cycle eventually leading to their own encysting stage.
\end{abstract}

Keywords: Stentor coeruleus; Stentor X; Encysted cells; micronuclei; Stentor media
Received Date: January 12, 2017

Accepted Date: January 24, 2017

Published Date: January 31, 2017

Citation: Scherlag, B.J. The Life Cycle of Dwarf Stentors. (2017) Lett Health Biol Sci 2(1): 45- 47.

DOI: $10.15436 / 2475-6245.17 .012$

\section{Introduction}

Unicellular animals of the genus Stentor have been the subject of study dating back to the $19^{\text {th }}$ century ${ }^{[1]}$. These organisms have been shown to regenerate entirely from surgically segmented sections ${ }^{[2]}$. More recent studies have ascribed other unusual abilities of the non-neural single cell organisms, including potential learning under controlled circumstances ${ }^{[3]}$.

In the present study, we discovered a previously unreported dwarf variety of actively mobile Stentor coeruleus that derived from progenitor micronuclei shed from the much larger, well known varieties that were allowed to encyst by evaporation and rehydration with sterile Stentor media in deep well slides.

\section{Methods}

Deep well slides (Figure 1, $\mathrm{n}=10$ ) were initially uncovered so that the well could be filled with Stentor culture solution. Both the deep well slides and Stentor cultures were obtained from Carolina Biological Supply Company (Burlington, NC, USA). The culture solutions were examined microscopically before and after the solutions were allowed to evaporate in the deep well slides (uncovered) for 24 hours. Stentor media obtained from Carolina Biological Supply Company was prepared from pellets dissolved in 1 liter of deionized water. After being brought to a boil the solution was decanted into $200 \mathrm{cc}$ jars and set aside to cool. After 24 hours, all uncovered wells with evaporated contents were filled with Stentor media solution and sealed with their respective plastic covers. Each deep well was examined microscopically for the first few hours and daily for the next 10 days. For controls, ten deep well slides were filled with sterile Stentor media and examined with the same schedule as described for the experimental group.

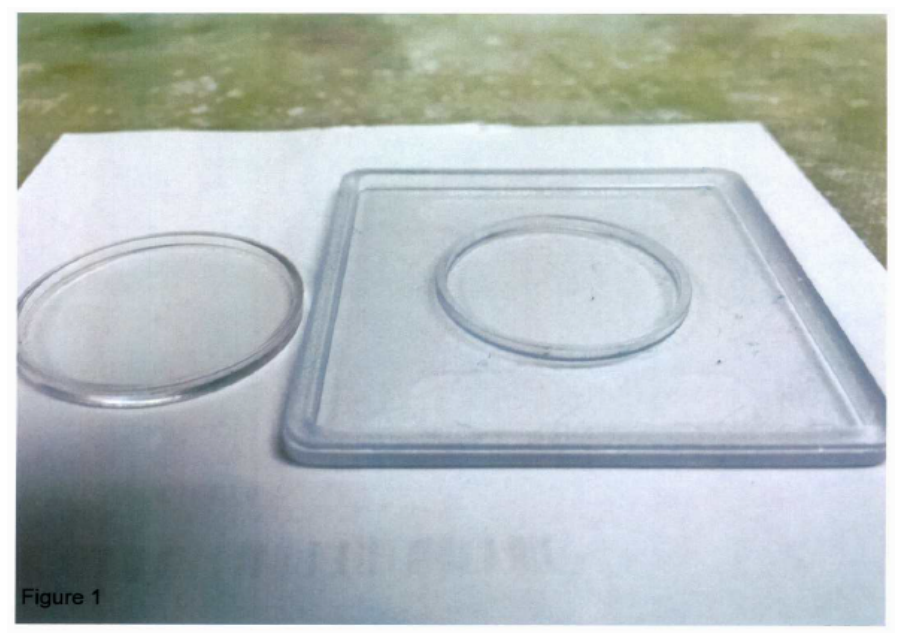

Figure 1: The uncovered deep well slide is shown at the right and the plastic sealing cover at the left.

Copyrights: (C) 2017 Scherlag, B.J. This is an Open access article distributed under the terms of Creative Commons Attribution 4.0 International License. 


\section{Statistical Analysis}

After 24 hours the number of deep well slides that showed dwarf "swimmers' and/or the presence of stationary cells with internal circulation, "progenitors." (See below) were compared with those in whom these features were not observed. Chi-square analysis (2X2 contingency table) was used to calculate the presence or absence of swimmers and progenitors followed by a Yates and Exact ad hoc test. A p value of $\leq 0.05$ was considered significant.

\section{Results}

(Figure 2 A-D) shows the various forms of large Stentors found in the initial culture solution placed in the deep well slides. These different ciliated varieties of Stentor coeruleus range in size from 500 to 1000 microns. They also showed differences as either sessile organisms, i.e. attached to vegetation (B and C) or as fast or slow free swimming forms (A and D, respectively).
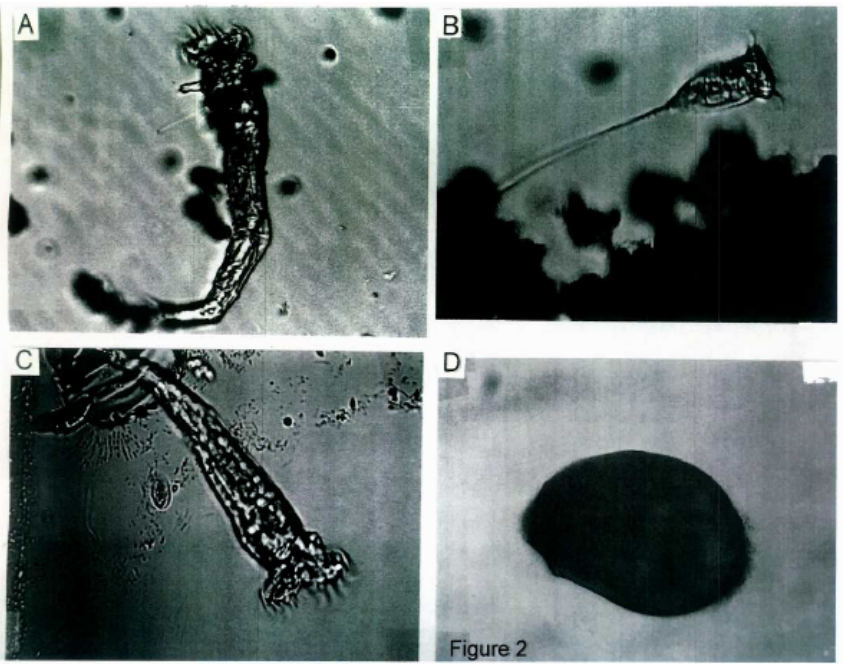

Figure 2: The different forms of the large Stentors. (A) A free swimming Stentor. (B) Trumpet shaped Stentor with long stem or holdfast attaching to vegetation. (C) Sessile form similar to Stentor in A, but attached to vegetation. (D) Oblong shaped mobile form. Magnification $10 \mathrm{X}$

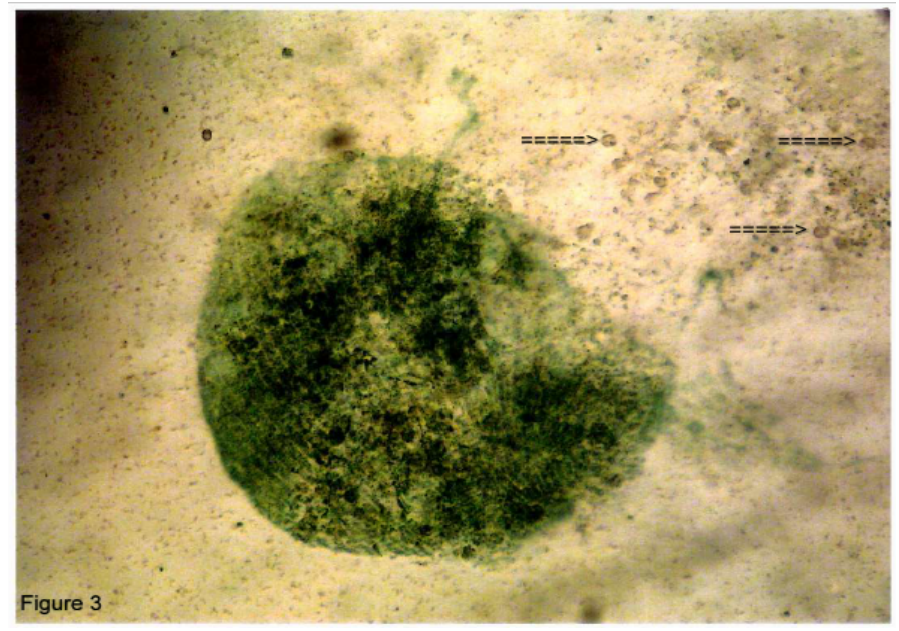

Figure 3: An encysted cell whose membrane has disrupted shedding micronuclei (arrows). Magnifications 10X.
After 24 hours of evaporation of the culture solution, these large forms of Stentor went into an encysted stage. Some of these cells showed disrupted membranes. Figure 3 illustrates such a cell with release of micronuclei (arrows).

At this time, the deep well slides were filled with the sterile Stentor media solution $(\mathrm{pH}$ 6.5) and the plastic covers were snapped into place sealing the contents within. Over the next 4 hours and successive days ( 10 days) the slides were examined microscopically. Specifically we searched for mobile dwarf cells and stationary cells with movement of internal inclusions. The former ranged in size from 30 - 45 microns whereas the latter were smaller ( 20 microns) showing circulating internal inclusions. Figure 4 (Vedio, shown in link: https://dl.dropboxusercontent.com/u/23485529/PICT0052.AVI, control/right click or copy $\&$ paste link into browser) shows a cell containing many inclusions in rapid motion. Within 10 - 20 minutes, the cell segmented and subsequently separated as 4 mobile individuals. In a small number of cases these active progenitor cells gave rise to only 2 "swimmer" which segmented and dispersed. It should be noted that the sequence and timing of these events were quite variable. Within 24 to 48 hours, it was possible to localise the progenitor call based on their size and spherical shape. Many of these cells were active in terms of movement of their internal inclusion, however many were relatively inactive and tented to be hose that proceeded to a more active segmenting and separating stage over a longer period of time. We also observed that at 48 hours there was the beginning of an overlap in appearance of similarly sized and spherical cells and the progenitor cells. These other cells types tended to show less dense internal inclusions and some lost their inclusion completely, becoming transparent circles. We surmised that these cells represented another stage in the Stentor's development or degeneration.

We followed the dwarf swimmers for the next few days and noted that their numbers increased considerably.At around ten days the number of dwarf swimmers markedly decreased and were replaced by large number of small rounded cells in which inclusions were mostly missing (Figure 5). None of the control deep well slides, containing sterile Stentor media, showed any evidence of dwarf swimmers or active or inactive progenitor cells under daily microscopic examination over a period of 10 days.

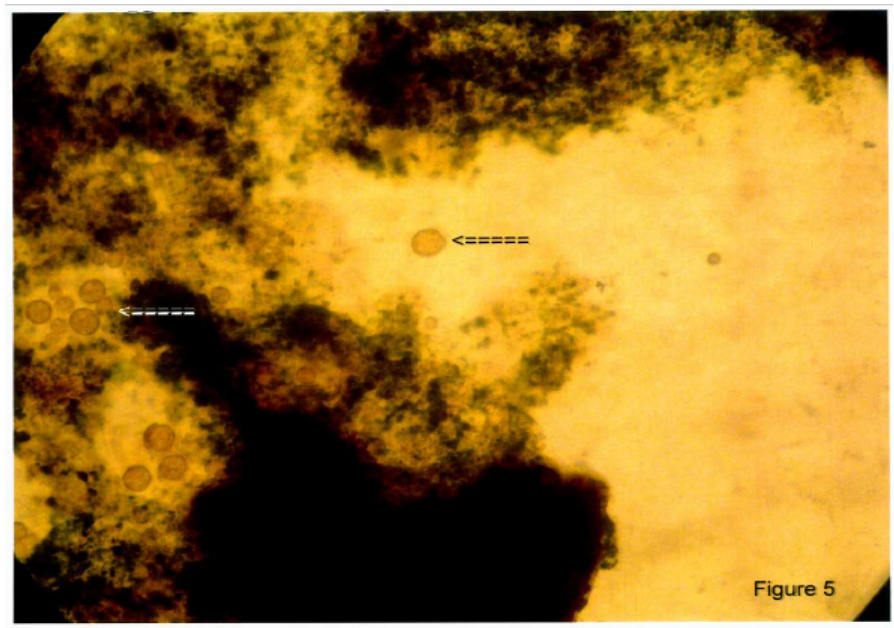

Figure 5: After 7 - 10 days there was a conversion of the free swimming dwarfs to encysted forms. Note the many spherical cells with loss of inclusions (arrows). Magnification 20X. 
A comparison between the sealed deep well slides $(\mathrm{n}=$ 10) containing the encysted Stentor coeruleus organisms in sterile Stentor media and those $(n=10)$ with only the sterile media found that $8 / 10$ of the former manifested both dwarf swimmers and dividing micronuclei; whereas none of the controls were positive in this regard, $\mathrm{p}=0.0007$ (two-tailed, Chi-square analysis, $2 \mathrm{X} 2$ contingency, Fisher Exact Test)

\section{Discussion}

\section{Major Findings}

We discovered a new dwarf form (30 - 45 microns) of Stentor coeruleus which derived from the division of micronuclei shed from disrupted encysted cells of the large forms (500 - 1000 microns) of Stentor. Some of these encysted cells showed disrupted membranes with shedding of their micronuclei. We refilled the deep well slides with sterile Stentor media and sealed the wells with their plastic covers. Within 24 hours, two cell forms, actively swimming dwarf cells and stationary micronuclei showing movement of their internal inclusions, appeared in the sterile Stentor media. Within minutes these micronuclei consistently divided into 2 or 4 new dwarfs which separated as mobile new mobile microorganisms. Over the next 7 - 10 days the numbers of swimmers increased and then decreased until only transparent encysted cells were seen within the sealed compartments.On the other hand, none of the control deep well slides containing only sterile Stentor media, showed any of these cell types.

\section{Background}

In the recent review (2014) of Stentor coeruleus, (ciliate phylum, class Heterotrichae) Slabodnick and Marshal[ ${ }^{[4]}$ describe the regenerative ${ }^{[2]}$ and learning abilities ${ }^{[3]}$ of this unusually large ( $\sim 1 \mathrm{~mm}$ long) single cell microorganism. Stentors have attracted the particular interest of zoologists and embryologists due to their ability to regenerate an entire organism from surgically cut sections. The most comprehensive studies of the Genus Stentor were presented by Johnson ${ }^{[1]}$ and Tartar ${ }^{[5]}$. Both mention the appearance of dwarf forms related to starvation in which the organisms self-cannibalize their internal content. The starved dwarfs are reduced in size ranging from 94 - 376 microns but regained their previous size when fed. These descriptions contrast with the dwarfs seen in the present study. These dwarfs ranged in size from 30 - 45 microns and derived from dividing micronuclei shed from the disrupted encysted cell of the much larger forms. Moreover they showed no characteristics of the larger forms specifically they lacked the characteristic blue green color and stripes [5, page 312]. In regard to the micronuclei, Johnson ${ }^{[1]}$ described them within the living Stentor bodies as minute (1 2 microns) but greatly enlarge at the time the large organisms show reproduction by fission or conjugation. It is difficult to determine from Johnson's description whether the micronuclei also undergo fission although he states, "As I have succeeded in finding them in mitotic division, there is no longer reason to doubt their micronuclear nature." In any event there is no mention of four dwarfs arising from a single enlarged micronucleus as we found in micronuclei as large as 25 microns, in diameter.

In their recent article, Slabodnick and Marshall[ ${ }^{[4]}$ have argued for more research using Stentor as a model for morphogenesis, wound healing and even for memory and learning on a single cell level. Our study confirms their view that Stentor research can yield new information in many life science fields.

\section{Limitations}

In the present experiments we tested the effect of introducing Stentor media into a deep well slide containing encysted Stentor coeruleus organisms. These agents with different $\mathrm{pH}$ levels were not tested nor other solutions to determine potential enhancing or inhibitory effects on dwarf cell or micronuclei activity. Tartar in $1957^{[6]}$ applied different solutions and chemicals to cultures of Stentor coeruleus to determine effects on parts of the cells whether they altered "cytoplasmic differentiation" and morphogenesis. It is interesting to note that Tartar found toxic effects on Stentor when he exposed them to sea water.

\section{Conclusions}

By instilling Stentor media into deep well slides containing encysted Stentor cells, there was a consistent appearance of mobile dwarf Stentors not seen in the original cultures. Four dwarfs derived from a single micronucleus released from encysted cells whose membranes had ruptured. Over a period of days, in a sealed compartment, these dwarfs increased in number then decreased eventually encysting themselves by 7 to 10 days.

Acknowledgement: The author thanks Branden Pannel for his assistance in the preparation of the manuscript for submission.

Funding Source: Supported by an unrestricted grant from the Helen and Wilton Webster Foundation through the Oklahoma Research foundation

\section{Conflict of Interest: None declared.}

\section{References}

1. Johnson, H.P. A contribution to the morphology and biology of the Stentors. (1893) J of Morphol 8(3): 467-562.

2. Lille, F.R. On the smallest part of Stentor capable of regeneration. (1896) J of Morphol 12(1): 239-249.

3. Hinkle, D.J., Wood, D.C. Is tube-escape learning by protozoa associative learning? (1994) BehavNeurosci 108(1): 94-99.

4. Slabodnick, M.M., Marshall, W.F. Stentor coeruleus. (2014) Current Biology 24(17): R783-R784.

5. Tartar, V. The Biology of Stentor. International series of Monographs on Pure and applied Biology. (1961) Pergamon Press New York.

6. Tartar, V. Reactions of Stentor coeruleus to certain substances added to the medium. (1957) Exptl Cell Research 13(2): 317-332. 